
\title{
FUNCTIONAL AND CLINICAL IMPLICATIONS OF THE VASCULAR ANATOMY OF NERVE
}

\author{
By Michael J. Blunt, M.B., B.S. \\ Department of Anatomy, St. Bartholomew's Hospital Medical College
}

Our knowledge of the anatomy of the blood supply of nerves has long withstood a dignified antiquity. Even before the publication of Harvey's classic paper on the circulation of the blood the Belgian anatomist, van der Spieghel (1627), wrote of the vasa nervorum in man, 'It is ridiculous to think, as Aristotle stated in his account of the body, that there should be any mucous-like, whitish and glutinous substance around the nerves, from which the nerves themselves spring and from which they are nourished, when their nourishment may be obtained from these vessels.' Ruysch (I 701), von Haller (1752) and Schmidel (I755) were all well aware of the presence of blood vessels to nerves and of their potential significance, and Boerhaave ( 1762 ) considered that knowledge of the blood supply of nerves would pave the way for the understanding of many of their diseases, 'so that it by no means is to be placed amongst the anatomical minutiae to be excluded altogether from the attention of the practitioner.'

In I768 Isenflamm and Doerffler published what appears to have been the first work devoted exclusively to the blood supply of nerves, a paper entitled De Vasis Nervorum. They had demonstrated fine vascular branches in the sheaths of peripheral nerves, of which they observed ' filling the latter (vessels) with coloured wax makes them elegantly conspicuous and gives a likeness of a painted vascular net enveloping the nerve.' They were also familiar with the intrinsic vascular plexuses which ramify in the endoneurial and perineurial sheaths of peripheral nerve trunks, and which were described in more detail by Ranvier (1878). He established that they were for the most part composed of longitudinally disposed vessels between which there were frequent transverse anastomoses; the plexuses were both intrafascicular and perifascicular in distribution.

Quénu and Lejars (1890, 1892 and 1894) paid particular attention to the numerous anastomoses between adjacent vasa nervorum, both on the surfaces of nerve trunks in the epineurium, and in their substance; they considered that the presence of these anastomoses would render a total intere ruption of the blood supply extremely unlikelyo The intercommunications of the veins draining. nerve trunks were considered even more consu spicuous than were those of the arteries of supply $y_{\omega}^{\omega}$ and particular significance was attached to the fac: that they tended to open into venous tributarieg from muscles, as venous return would thus beg accelerated by muscular contraction.

After the end of the Igth century anatomica $\Gamma_{n}$ investigations into the blood supply of nerves were limited to the detailed vascular topography of number of different nerve trunks and to the elaboration of points of detail, but no new bagic principles emerged to add to those previougly established.

The inferences regarding the relative importapice of the regional nutrient vessels and of the intrinsis longitudinal vascular plexuses of nerve trunk which may be drawn from data on their vasculañ anatomy must, of necessity, be very limited, fon the effectiveness of intercommunications betweer $\vec{b}$ adjacent groups of vasa nervorum cannot be judged $B$ on anatomical grounds alone. The evidence re lating to the problem of whether or not the blood? supply of nerves is functionally segmental has comes from other sources, partly from direct and indirect. experimental approaches and partly from in $\frac{5}{7}$ vestigations into the influence of regional bloot supply on the degeneration and regeneration of nerve fibres. The main findings from each of these sources are here considered in turn and the present status of the vasa nervorum and the clinical im $\rightarrow$ plications of their anatomy are discussed.

\section{General Experimental Evidence}

In general, two types of experiment have been used to study the blood supply of nerves. In thosew of the first type a segment of nerve trunk has beep subjected to pressure, the results of which have been attributed to ischaemia; in the second typeD direct interruption of nutrient vessels has been effected. Because it is likely that compression? results in the grossest form of ischaemia to which 
a length of nerve trunk may be subjected, the results of this type of experiment are considered first.

Lewis, Pickering and Rothschild (I93I) were the earliest investigators to study the effects of pressure applied to nerve trunk specifically in regard to the possibility of ischaemia being the agent responsible for the results obtained. From experiments in which pneumatic cuffs were applied to human limbs, they brought evidence to show that the paralysis and anaesthesia so produced were due to ischaemia of the compressed segments of nerves under the cuff. Their results were criticized by Bishop, Heinbecker and O'Leary (1933) and by Bentley and Schlapp (1943b). The former investigators showed that increasing degrees of pressure applied to nerve trunks could lead to increasing degrees of conduction block; the latter authors demonstrated that the block to conduction produced by direct compression of a nerve trunk by pneumatic pressure was, under the conditions of their experiments, maximal at the margins of the compressed segment. Both of these groups of investigators therefore considered that the conduction block was due to local deformation of nerve fibres and that circulatory failure was not an important factor in its production. However, DennyBrown and Brenner (I944) showed that the compartmented structure of the sciatic nerve of the cat could protect some of its longitudinal vessels from external pressures and concluded that the variability of the results of nerve compression at different pressures was related to the different degrees of ischaemia so produced. Causey and Palmer (1949) used a specially designed mercury pressure box to study the effects of pressure on nerves without producing uneven deformation at the margins of the compressed segment. At all pressures above $150 \mathrm{~mm}$. $\mathrm{Hg}$ a block to conduction ensued, but when air and not mercury was used as a compressing agent there was no block to conduction. It was concluded that, in acute experiments, the failure to conduct an impulse after local pressure on a nerve trunk was caused by anoxia of the compressed segment. The hypothesis that the effects of pressure on nerve trunk are due to ischaemia and subsequent anoxia has, therefore, been substantiated. Since failure of conduction in a fascicular nerve like the sciatic may be long delayed because of the residual patency of a few vessels in the central part of the nerve, the evidence suggests that a fairly severe degree of ischaemia must be produced before failure of nerve conduction follows.

Bentley and Schlapp (1943a) found that a fairly extensive devascularization of the sciatic nerve in experimental animals, produced by mobilization and division of the nerve, resulted in a block to conduction. Causey and Stratmann (1953) found that deliberate removal of as much as possible of the blood supply to one end of the sciatic nerve of the rabbit produced marked alterations in the course of recovery of the nerve fibres from fatigue after high - frequency stimulation. From the severity of the devascularization employed in the experiments of Bentley and Schlapp and Causey and Stratmann it might be assumed that the regional vessels have but slight significance in the maintenance of an adequate blood supply to a segment of nerve trunk. There is, however, evidence that such is not the case and recent work has shown that, because of the high degree of ischaemia needed to produce measurable impairment of nerve conduction, the results of electrophysiological experiments probably constitute an inadequate criterion by which alone to assess the importance of vasa nervorum. Blunt and Stratton (1956) used the clearance of radioactive sodium injected into the sciatic nerve of the rabbit as an index by which to assess the relative importance of regional neureal vessels and intrinsic vascular plexuses. It was thereby shown that the vasa nervorum to the lower end of this nerve were more effective sources of blood supply than were the intrinsic plexuses of the nerve trunk. Recovery experiments, however, demonstrated a striking early compensation for the effects of ligature of these segmental vasa nervorum; this was effected by the neural intrinsic longitudinal vessels and was complete, under the conditions of the experiments, by the end of Io days. Despite this early recovery, new growth of regional vessels took place and by the end of 20 days the newly formed vessels made a substantial contribution to the blood supply of the nerve on a segmental basis.

\section{Influence of Blood Supply Úpon Degeneration and Regeneration of Nerve Fibres}

Denny-Brown and Brenner investigated the histological changes in nerve after ligation of regional vessels. After tying the regional vessels to the sciatic nerve of cats no defect in conduction was demonstrated, but histological examination two weeks later revealed patchy areas of swelling of the axis cylinders with vacuolation of their myelin sheaths. The authors claimed that this type of lesion is characteristic of ischaemic damage and forms a distinctive neuropathological reaction. The changes they observed in the nerve fibres were not followed over long periods of time. However, because of the rapidity with which a collateral circulation develops after ligature of vasa nervorum, it is reasonable to conclude that the degenerative changes noted were not, in the conditions of these experiments, progressive.

The work of Adams (1943) and Durward (1948) has revealed that actual fibre degeneration, as 
revealed by the Marchi technique, can only be produced after the most extensive devascularization of nerve trunks and probably only after damage to regional vasa nervorum is reinforced by occlusion of longitudinal epineurial vessels. Nevertheless, it must be remembered that a degree of vascular occlusion insufficient to result in conduction block or in complete fibre degeneration may yet be followed by recognizable histological changes in the nerve fibres.

The influence of the regional (segmental) blood supply of nerves upon fibre regeneration rates after experimental crush injuries has been studied by Bacsich and Wyburn (1945). They found no statistically significant difference between the mean rates of axonal advance in nerves which were simply crushed and in nerves which were both crushed and devascularized. However, it must be observed that, in each experimental animal, there was consistently slower regeneration in the crushed and devascularized nerve than in the crushed control nerve of the opposite side.

In consequence of the results of some of the experiments reviewed above, there has been a rather general tendency to under-estimate the importance of vasa nervorum. It is obviously important to know that, in the experimental animal, much of the blood supply of a nerve may be sacrificed without producing true fibre degeneration and without interfering with fibre regeneration to a statistically significant extent. Of more significance, however, is the problem of whether the overall results of local devascularization can result in ischaemic damage to peripheral nerves to an extent which is of clinical importance. This problem has not yet been answered by experimental procedure, but there is evidence from pathology to indicate that such ischaemic damage is, in fact, a not infrequent complication of neurovascular injury.

The nerve lesions in patients with Volkmann's ischaemic contracture have been described by Holmes, Highet and Seddon (1944) and consist of a range of pathological changes varying from axon and myelin sheath destruction, as in Wallerian degeneration, to a greatly increased amount of collagen in the endoneurium, sometimes amounting to complete collagenous replacement of nerve bundles. In regions where ischaemia had been particularly severe from the onset a coagulative necrosis of the nerve fibres was sometimes seen. Such ischaemic damage to nerve trunks results from a gross vascular deficiency severe enough to impair the nutrition of all the tissues in a limb; that localized vascular damage may cause nerve lesions alone has also been reported. Seddon and Holmes (1944) described a case in which there was ischaemic damage to the peripheral stump of a median nerve divided in the upper third of the forearm; the anterior interosseous artery had alsu been damaged. The median nerve was reduced a minute thread down to the level of the wrise? and histological examination revealed a massive increase in the collagen of the endoneurium, lead ing, in most areas, to complete obliteration of the Schwann tubes. Woodhall and Davis (195 reported two similar cases. In the first there hax been acute thrombosis of the arteria comes nergi ischiadici, and in the second a branch of the brachial artery to the median nerve had been blocked by thrombosis. In both instances massive collagenization of the distal segment of the divide nerve trunk had resulted.

The results of primary nerve suture are oftecis unsatisfactory. Blackwood and Holmes (1954). stated that in all the biopsy specimens which thege examined because of unsatisfactory results from primary suture, the nerve stumps showed a degres of fibrosis sufficient to preclude satisfactory r\& generation. They considered that the changes observed were probably due to ischaemia. Woodhall and Davis, from a study of nerve biopsieq taken from divided main nerve trunks at the timf of definitive operations, found similar changes i many cases. They concluded that 'excessive mobilization of nerve segments, and in particglar the distal segment, with injudicious sacrifice of remaining lateral nutrient vessels, may init解施 intraneural changes compatible with those scribed in ischaemia.'

The evidence from pathology indicates, there fore, that severe ischaemic changes may urg doubtedly occur as a result of local interferene with the blood supply of peripheral nerves. It $\overline{6}$ not possible to estimate the relative results of injury to the segmental vessels alone and to the intrinsic vascular plexuses. However, after per? pheral nerve lesions which are disruptive of com tinuity and which involve interruption of the intr\% neural vascular plexuses, an adequate blood supp $\bar{B}$ to the nerve trunk below the level of the lesion $\frac{3 i s}{45}$ largely dependent upon the integrity of its seg mental nutrient vessels.

\section{Conclusions}

It may be concluded from the results so fir reported, and which are briefly reviewed abov\& that the blood supply of peripheral nerve trunks is anatomically segmental and is derived from a serißß of separate vasa nervorum. This basically seg mental pattern is, however, to a large extent masked by the epineurial anastomoses between adjacet vasa nervorum and by the longitudinally orientated intrinsic vascular plexuses of the nerves, through the medium of which also the adjacent regiongl vessels are linked to one another. This anatomiced 
pattern of vascularization is reflected in the results of ligature of vasa nervorum, for it has been shown by studying the clearance of radioactive sodium that a single group of regional vessels dominates the circulation in its own length of nerve trunk. The importance of the epineurial anastomoses, the intrinsic longitudinal plexuses and the free anastomosis which these afford between adjacent segmental vasa nervorum finds expression, however, in the rapid establishment of a compensatory collateral circulation after ligature of the segmental vessels. Damage to segmental vessels alone does not result in more than relatively minor histological changes.

If damage to regional neural vessels is associated with destruction of the epineurial anastomoses or occlusion of the intrinsic longitudinal plexuses it seems highly likely that true fibre degeneration results, though a limited number only of the nerve fibres may so suffer.

It is when the intrinsic vascular plexuses of a nerve have been interrupted by injury that the problems of most obvious practical significance arise, for a balance must be reached between the amount of nerve mobilization necessary to secure adequate relief of tension before suture of a divided nerve, and a degree of mobilization likely to diminish the somewhat precarious blood supply of the distal stump to a level at which ischaemic changes preclude satisfactory regeneration. That mobilization should be adequate before secondary suture has been shown by Bowden (195I and personal communication). She compared two series of low ulnar nerve sutures and the results were significantly inferior in a group where mobilization was limited and the nerve ends were approximated by acute flexion of the wrist to those in a group where more extensive mobilization was carried out. Possibly the advantages of free mobilization may be combined with those of an adequate blood supply to both proximal and distal nerve segments if the mobilization is associated with careful preservation of local nutrient arteries, particularly those of the distal segment. Advantage may also be gained by concentrating, where the circumstances permit, on mobilization of the prox mal segments of divided nerves, the distal segments being relatively undisturbed. Such problems of treatment as have been briefly considered cannot be lightly dismissed, for it seems quite clear that occlusion of the regional vessels, either by tho original injury or by the operative mobilization of nerve segments prior to suture, may result in pro found histological changes sufficient to precludes regeneration of the severed nerve fibres because of collagenous obliteration of their Schwann tubes. .

The author is very grateful to Professor R. E. MF Bowden and Professor A. J. E. Cave for their helpo ful advice during the preparation of this paper.

\section{BIBLIOGRAPHY}

ADAMS, W. E. (1943), f. Anat. (Lond.), 77, 243.

BACSICH, P., and WYBURN, G. M. (1945), F. Anat. (Lond.D্ক BENTLEY, F. H., and SCHLAPP, W. (1943a), f. Physiol., 102, 6 尺 BENTLEY, F. H., and SCHLAPP, W. (1943b), f. Physiol., roz BISHOP, G. H., HEINBECKER, P., and O'LEARY, J. L. (19332. Amer. $₹$. Physiol., 106, 647 .

BLACKWOOOD, W,' and HOLMES, W. (1954), in ' PeripheralT Nerve Injuries,' Medical Research Council, London.

BLUNT, M. J., and STRATTON, K. (1956a), $\mathcal{F}$. Anat. (Lond.

BLU'NT, M. J., and STRATTON, K. (1956b), f. Anat. (Lond. 迹 BOERHAAVE, H. (1 762), 'Praelectiones Academicae de Mofis Nervorum, Frankfurt.

BOWDEN, R. E. M. (195I), Ann. Roy. Coll. Surg., 8, 366.

CAUSEY, G., and PALMER, E. (1949), $\mathcal{F}$. Physiol., 109, 220.

CAUSEY, G., and STRATMANN, C. J. (1953), $\mathcal{F}$. Physiol,

DENNŸ-BROWN, D., and BRENNER, C. (1944), Arch. Nêro Psychiat., Chicago, 51, I.

DURWARD, A. (1948), Postgrad. med. F., 24, 11 .

VON HALLER, A. (1752), Iconum Anatomicarum, 5, Gottingen. HOLMES, W., HIGHET, W. B., and SEDDON, H. J. (1944 Brit. $\mathcal{F}$. Surg., 32, 250 .

ISENFLAMM, J. F and DOERFFLER, J. F. (1768),

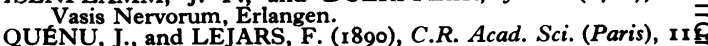

QUER. QUENU, J., and LEJARS, F. (I894), 'Etudes sur le Syst èn Circulatoire,' Paris.

RANVIER, M. L. (1878), ' Système Nerveux, I ,' Paris. RCHMIDEL, C. C.' (1755), quoted by Isenflamm and Doerffer. 3 SCHMIDEL, C. C. (1755), quoted by Isenflamm and Doerffer. SEDDON, H. J., and HOLMES, W. (1944), Brit. F. Surg., 32, 38 WOODHALL, B., and DAVIS, C. (1950), Ұ. Neuropath. exp Neurol., 9, 335 .

\section{RUTHIN CASTLE, NORTH WALES}

A Clinic for the diagnosis and treatment of Internal Diseases (except Mental or Infectious Diseases). The Clinic is provided with a staff of doctors, technicians and nurses.

The surroundings are beautiful. The climate is mild. There is central heating throughout. The annual rainfall is 30.5 inches, that is, less than the average for England.

The fees are inclusive and vary according to the room occupied.

For particulars apply to THE SECRETARY, Ruthin Castle, North Walas. 